\title{
Distribution of cases of systemic lupus erythematosus at time of first symptom in an urban area
}

\author{
N D Hopkinson, K R Muir, M A Oliver, M Doherty, R J Powell
}

Department of Immunology, Queen's Medical Centre,

Nottingham, United Kingdom N D Hopkinson

Department of Public Health Medicine and Epidemiology

K R Muir

School of Geography, The University of Birmingham, Birmingham B15 2TT, United Kingdom M A Oliver

Rheumatology Unit, City Hospital, Nottingham, United Kingdom M Doherty

Correspondence to: Dr N Hopkinson Department of Rheumatology and Rehabilitation, Royal Bournemouth Hospital, Castle Lane East, Bournemouth BH7 7DW, United Kingdom.

Accepted for publication 19 June 1995

\begin{abstract}
Objectives-To determine the geographical distribution of cases of systemic lupus erythematosus (SLE) in a defined geographical area in the East Midlands, UK, and, in particular, to search for spatial variation in cases that may implicate the role of environmental factors in SLE aetiology.

Methods-Six methods of case ascertainment were used. The postcode of the patient's domicile at time of first definite symptom of SLE was used for analysis which included case mapping, probability mapping by electoral ward, and variogram analysis.

Results-The study area population of 613700 contained 200 SLE patients, 188 of whom experienced their first symptom whilst residing in the area. Case mapping revealed 12 SLE patients residing within an area of one square mile, including four men and six patients with RNP antibodies. The use of probability mapping showed five wards in close proximity to each other to have a greater number of SLE cases than would be expected by chance $(p<0 \cdot 1)$. The 'cluster' of patients seen on the case map fell into two wards which showed a significant excess of cases only when combined $(p=0 \cdot 006)$. The variogram of the incidence rates for each ward did not confirm any structure or pattern to the distribution of cases for the whole area.

Conclusions-Some areas have a greater than expected prevalence of SLE. The normal result from variogram analysis suggests that the cause(s) for these excess number of cases does not have an effect across the whole study area.
\end{abstract}

(Ann Rheum Dis 1995; 54: 891-895)

There is marked variation in the geographic distribution of many human diseases, and Snow's investigation of the clustering of cases of cholera around a water pump in London in $1848^{1}$ led to the subsequent identification of its causative agent. Similarly, Burkitt's geographical analysis of the lymphoma that now bears his name has given insight into its possible causes. ${ }^{2}$ More recently, the distribution of other cancers, particularly leukaemia, has been scrutinised using a variety of techniques ${ }^{3}{ }^{4}$ and such studies have led to hypotheses relating environmental factors to the occurrence of a disease.

Systemic lupus erythematosus (SLE) is an uncommon disease (prevalence, $24 \cdot 7 / 100000^{5}$ ) in which genetic and environmental factors are likely to be important. Genetic influences are notable in complement deficiencies ${ }^{6}$ and in twin concordance studies. ${ }^{7}$ The importance of environmental effects has been argued on the basis of geographical differences in prevalence rates between so-called common genetic stock, for example black people in Africa and those in North America ${ }^{5-10}$ and, similarly, Chinese groups living in Malaysia, Hawaii, and North America. ${ }^{11}{ }^{12}$ It is well established also that a number of drugs, for example hydrallazine and procainamide, can induce a disease which is not dissimilar to idiopathic SLE. ${ }^{13}$ However, the spatial distribution of SLE has received little attention. ${ }^{1415}$

The geographical distribution of SLE in the Nottingham Health District, UK, has been studied to assess associations with environmental factors. Methods of data analysis applied to cases of SLE included probability mapping, ${ }^{16}$ and variogram analysis.

\section{Methods}

The study was approved by the local ethics committee. The study area was that of the Nottingham Health District, which is served by two main hospitals, the Queen's Medical Centre (QMC) and Nottingham City Hospital. An attempt was made to identify all patients with SLE residing in this area during the period 1 May 1989-30 April 1990.

IDENTIFICATION OF PATIENTS

Six principal methods of ascertainment were used, details of which have been published previously. ${ }^{5} 17$ From these sources, patients with definite or possible SLE who resided in the study area were contacted and invited to attend the hospital to consult one of the investigators $(\mathrm{NH})$.

\section{PATIENT INTERVIEW}

At interview the diagnosis of SLE was confirmed, or refuted, based on the principles of Fries and Holman, ${ }^{18}$ using evidence of multisystem disease, immunological abnormalities (for example positive ANA with or 
without antibodies to dsDNA) and in the absence of a more appropriate diagnosis. For this study patients were included regardless of the number of American Rheumatism Association (ARA) criteria that they fulfilled. ${ }^{19}$ The time of the first definite symptom of SLE was identified retrospectively and the patient's address and postcode at that time were recorded. If the patient had lived there for less than six months, their previous address was used for analysis.

THE STUDY AREA

The defined area approximated to the metropolitan community of Greater Nottingham, which consists of the districts of Nottingham, Broxtowe, Gedling, Rushcliffe, and the Hucknall wards of Ashfield. ${ }^{5}$ Based on 1988 mid-year estimates, the population of the area was 613700 (300600 males, 313100 females). ${ }^{20}$ The age and sex distrubution of this local population accords with that of the UK. ${ }^{21}$ All the major social classes are represented, with a distribution similar to that in the whole of England and Wales. The main industries in the area include mining, quarrying, and the manufacture of textiles, chemicals, and tobacco. ${ }^{22}$

GEOGRAPHICAL DISTRIBUTION OF SLE

The home of each patient with SLE was referenced by the postcode, according to the above criteria, and from this the geographical coordinates were derived. The electoral ward to which each case belonged was also derived from the postcode ${ }^{316}$ and this was referenced by the coordinates of the ward centroid (the population weighted centre). The cases were plotted on a map of Nottingham Health District to examine the spatial distribution. The rate of incidence of SLE for each ward was defined as the number of cases divided by the population in that ward:

$$
F\left(x_{i}\right)=L\left(x_{i}\right) / n\left(x_{i}\right)
$$

where $F\left(x_{i}\right)=$ rate of incidence or frequency, $L\left(x_{i}\right)=$ number of cases in ward $i$, and $x_{i}$ denotes the coordinates of the centroids of the wards.

We used two analytical approaches to assess potential patterns in the distribution of cases. These were chosen from a range of techniques in order to identify different types of pattern that may be present, as described further below.

\section{Poisson probability test}

The Poisson analysis is based on the concept that if cases are distributed independently of one another in geographical space with an intensity related only to population density, then the difference between the observed and expected number of cases will follow a Poisson distribution. The aim is to identify anomalies in the distribution of cases that might indicate real differences in the risk of developing the disease from area to area.
The expected number of cases of SLE in each electoral ward was calculated assuming a uniform distribution of SLE throughout the area. The incidence rates for each ward were tested against their expected number using a cumulative Poisson probability test and the wards ranked according to techniques described previously. ${ }^{316}$ To highlight those wards at the top of this table, a $p$ value of $0 \cdot 1$ was arbitrarily taken as being significant. This is superior to a simple observed:expected ratio because it allows for ward population. The $p$ values quoted are one sided.

\section{Geostatistical analysis}

Theory: variogram analysis. The variogram is the central tool of geostatistics, and is a set of analytical procedures based on the regionalised variable theory, developed by Matheron. ${ }^{23}{ }^{24} \mathrm{It}$ has been used widely for describing and estimating the spatial distribution of metals, diamonds, soil properties, and atmospheric pollutants. Geostatistics provides a statistical tool for describing variation across the Earth's surface. A recent development of the method has been applied to geographical patterns of rare diseases such as the analysis of the risk of childhood cancer, in which it has proved valuable. ${ }^{25}$ However, the technique is relatively insensitive to point clusters.

The variogram. Following geostatistical convention, the rate of incidence is treated as a variable, $Z(x)$, distributed in space in which $x$ denotes the coordinates, $x 1$ and $x 2$, for the two dimensions at the centre (or centroid) of each ward. Where a variable is spatially dependent, the variance of any pair of values depends on the separation in both distance and direction between them-the 'lag', denoted by the vector $h$. The variance of the differences between any two centroids a given distance apart is known as the semivariance, $g$, because it is the variance per site when the sites are considered in pairs. It is defined by:

$$
g(h)=1 / 2 E\left\{(Z(x)-Z(x+h))^{2}\right\}
$$

where $Z(x)$ and $Z(x+h)$ are the value of the property at any two places, $x$ and $x+h$, separated by $h$, and $E$ denotes the expectation. The function $g(h)$ that relates the variance to the lag is the variogram.

\section{Results}

On 1 May 1989, 163 people with SLE were residing in the area and a further 37 presented between that date and 30 April 1990. Of these 200 cases (177 women, 23 men), 188 experienced their first symptom while residing in Nottingham and were therefore suitable for inclusion in the geographical analysis.

\section{CASE MAP OF NOTTINGHAM}

The map of cases for Nottingham, plotted according to the individuals' domicile at the time of first symptom of SLE, independent of ARA criteria, showed that they were not distributed uniformly (fig 1). One area to the 


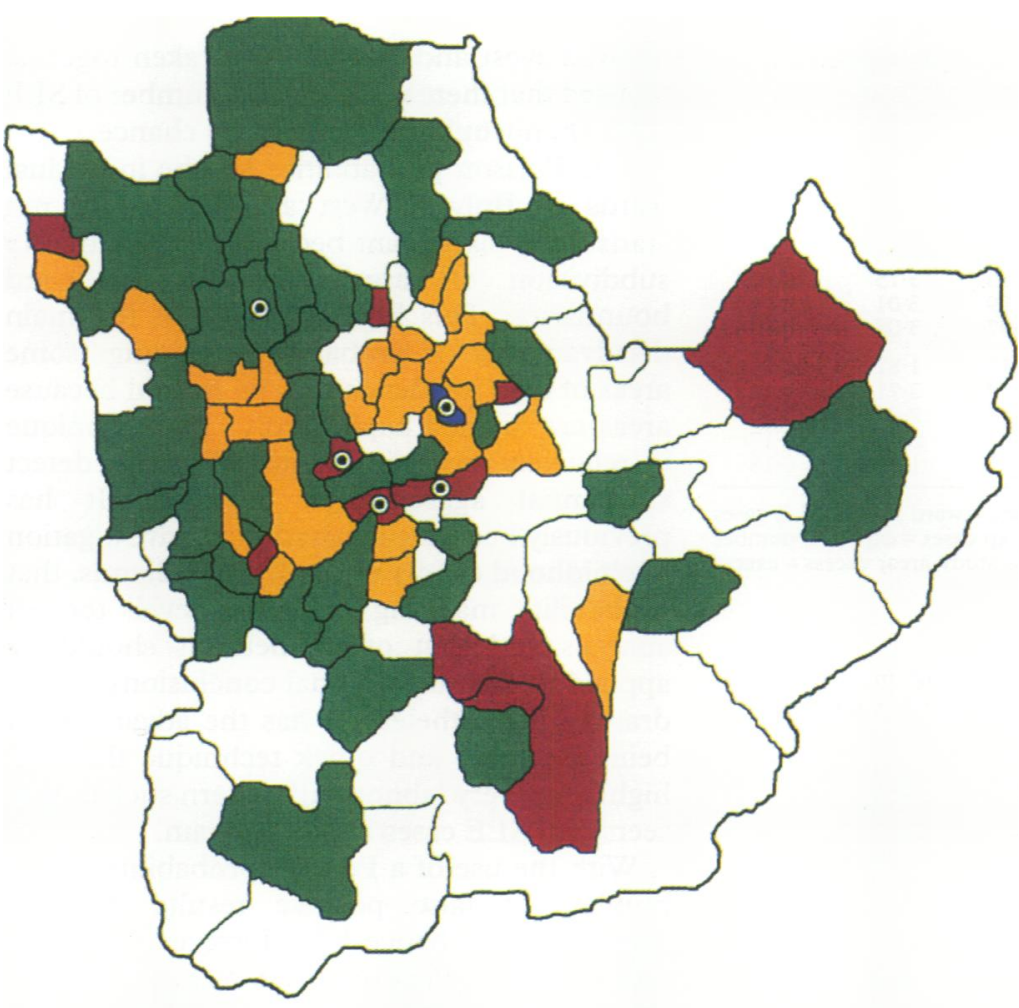

$0: E>3$

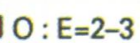

$O: E=1-2$

\section{$\square \mathrm{O}: \mathrm{E}=0-1 . \quad \square \mathrm{O}: \mathrm{E}=0$}

Figure 1 Map of study area showing observed to expected (O:E) ratio of cases of systemic lupus erythematosus by electoral ward. $\odot=$ Wards with a Poisson probability of $p<0 \cdot 1$. including three with antibodies to RNP, were living within one mile of this central area. None of these patients was related, knew each other, or had the same occupation. Onset of the patients' symptoms occurred over the period 1975-89. Table 1 shows the clinical details of the 12 patients in this area, who were referred by different General Practitioners.

CUMULATIVE POISSON ANALYSIS

Using the study area population of 613700 the rate of SLE patients presenting with their first symptom of SLE was $188 / 613700 \times$ $100000=30 \cdot 63 / 100000$ population.

Assuming an even spread of SLE across the population of the study area, the expected and observed number of cases per electoral ward was derived. Of 104 wards, 34 had no cases of SLE, 23 had one case and 47 had more than one case. Table 2 shows the wards with the smallest values of $p$. Clearly, this represents the top of a long list of electoral wards, the majority of which show a rate of occurrence of SLE similar to that expected by chance. At the bottom of the list are some wards that showed no cases of SLE despite being well populated. Using the incidence rate of $30 \cdot 63 / 100000$, all wards with a population of 3265 or more would be expected to have at least one case of SLE. Table 3 shows the 16 wards in the study area with larger populations than this, but with no case of SLE. Figure 1 shows the ratios of the observed to the expected number of cases of SLE for each electoral ward.

Using a Poisson probability of less than 0.01 as being significant, two wards had a greater than expected number of SLE cases: Carlton Hill in the Borough of Gedling and Lenton in the Borough of Nottingham. In figure 1, the geographical distribution of wards with a Poisson probability of $p<0 \cdot 1$ is highlighted by a circular symbol. Two such wards, Trent and Bridge, are adjacent to each other; and a further two, Lenton and Carlton Hill, are within two wards of these. Further away is the ward of Bulwell West. The cluster of 12 cases detailed above was contained within the wards of Bulwell West and Bulwell East. Neither ward on its own (table 2) showed an excess of cases using conventional statistical significance, but a significant excess in the number of SLE cases (observed 12, expected $6.94 ; p=0.006)$ was apparent when these two wards were combined.

Population pyramids for the five areas with a $p$ value less than $0 \cdot 1$ compared with the study area as a whole, have been constructed (data not shown). Bulwell West has a considerably higher proportion of children aged 0-14 compared with the whole study population, which would reduce the expected at risk population for SLE, with consequent enhancement of the significance of the $p$ value. In this SLE cohort, the greatest incidence rates were observed in the 40-59 year age group, which was in fact under represented in these high incidence wards, again increasing the significance of their observation. 
Table 2 Mapping of cases of systemic lupus erythematosus (SLE) by place of residence at first symptom, according to electoral ward

\begin{tabular}{lrllllll}
\hline $\begin{array}{l}\text { Electoral } \\
\text { ward }\end{array}$ & Popn & $\begin{array}{l}\text { Obs } \\
\text { cases }\end{array}$ & $\begin{array}{l}\text { Rate } \\
\left(/ 10^{3}\right)\end{array}$ & $\begin{array}{l}\times \text { Area } \\
\text { rate }\end{array}$ & $\begin{array}{l}\text { Exp } \\
\text { cases }\end{array}$ & Excess & $p$ \\
\hline Carlton Hill & 6255 & 7 & 1.12 & 3.65 & 1.92 & 5.08 & 0.0036 \\
Lenton & 7535 & 7 & 0.93 & 3.03 & 2.31 & 4.69 & 0.0096 \\
Trent & 9300 & 6 & 0.65 & $2 \cdot 11$ & 2.85 & 3.15 & 0.068 \\
Bridge & 9765 & 6 & 0.61 & 2.01 & 2.99 & 3.01 & 0.083 \\
Bulwell W & 12965 & 7 & 0.54 & 1.76 & 3.97 & 3.03 & 0.098 \\
Oxclose & 3705 & 3 & 0.81 & 2.64 & 1.13 & 1.87 & 0.10 \\
Robin Hood & 10735 & 6 & 0.56 & 1.82 & 3.29 & 2.71 & 0.11 \\
Beeston C & 6310 & 4 & 0.63 & 2.07 & 1.93 & 2.07 & 0.13 \\
Bulwell E & 9690 & 5 & 0.52 & 1.68 & 2.97 & 2.03 & 0.17 \\
Hucknall W & 9820 & 5 & 0.51 & 1.66 & 3.01 & 1.99 & 0.18 \\
\hline
\end{tabular}

Popn = Population; Obs cases $=$ observed number of SLE cases; Rate $=$ ward rate of SLE cases per 1000 population; $\times$ Area rate $=$ ward rate/total study area rate; Exp cases $=$ expected number of SLE cases assuming an even spread of SLE per head across the study area; excess = excess of observed cases over expected cases.

Table 3 Wards in the study area without any case of systemic lupus erythematosus (SLE) despite having an expected number $>1$

\begin{tabular}{lllll}
\hline $\begin{array}{l}\text { Electoral } \\
\text { ward }\end{array}$ & Popn & $\begin{array}{l}\text { Obs } \\
\text { cases }\end{array}$ & $\begin{array}{l}\text { Exp } \\
\text { cases }\end{array}$ & $p$ \\
\hline Basford & 9655 & 0 & $2 \cdot 96$ & $0 \cdot 052$ \\
St Anns & 8875 & 0 & $2 \cdot 72$ & $0 \cdot 066$ \\
Bingham & 7490 & 0 & $2 \cdot 29$ & $0 \cdot 10$ \\
Calverton & 6635 & 0 & $2 \cdot 03$ & $0 \cdot 13$ \\
Kingswell & 5925 & 0 & $1 \cdot 81$ & $0 \cdot 16$ \\
Stapleford W & 5785 & 0 & $1 \cdot 77$ & $0 \cdot 17$ \\
Stapleford E & 5775 & 0 & $1 \cdot 77$ & $0 \cdot 17$ \\
Hucknall E & 5665 & 0 & $1 \cdot 74$ & $0 \cdot 18$ \\
Toton & 5570 & 0 & $1 \cdot 71$ & $0 \cdot 18$ \\
Bonington & 4910 & 0 & $1 \cdot 50$ & $0 \cdot 22$ \\
Melton & 4845 & 0 & $1 \cdot 48$ & $0 \cdot 23$ \\
Eastwood E & 4835 & 0 & $1 \cdot 48$ & $0 \cdot 23$ \\
Bestwood Park & 4535 & 0 & $1 \cdot 39$ & $0 \cdot 25$ \\
Malkin & 4430 & 0 & $1 \cdot 36$ & $0 \cdot 26$ \\
Burton Joyce & 3795 & 0 & $1 \cdot 16$ & $0 \cdot 31$ \\
Beeston C & 3540 & 0 & 1.08 & $0 \cdot 34$ \\
\hline
\end{tabular}

Popn = Population; Obs cases $=$ observed number of SLE cases; Rate $=$ ward rate of SLE cases per 1000 population; Exp cases $=$ expected number of SLE cases assuming an even spread of SLE per head across the study area.

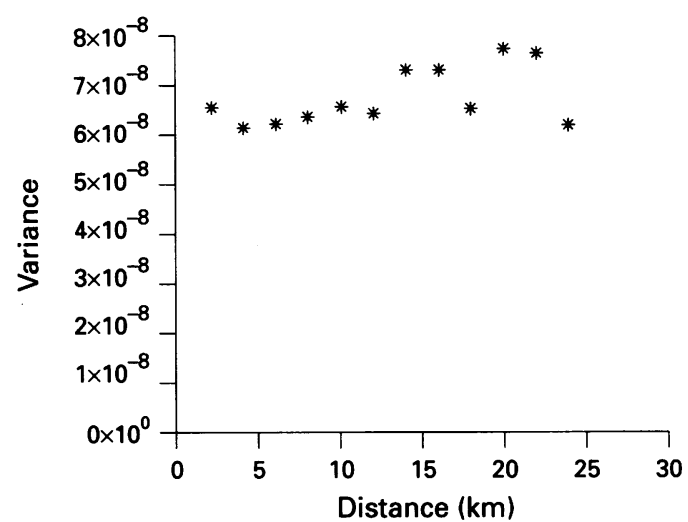

Figure 2 Variogram of frequency of cases of systemic lupus erythematosus in Nottinghamshire.

VARIOGRAM ANALYSIS

The variogram of the incidence rates for each ward suggested that there was no apparent structure or pattern for the defined study area (fig 2).

\section{Discussion}

When the place of residence of the patient at the time of their first symptom of SLE was mapped, the large number of cases in the Bulwell area and particularly the close proximity of the individuals led us to consider complex geographical analyses. The cumulative Poisson probability test for the wards of
Bulwell West and Bulwell East taken together showed that there was a greater number of SLE cases than would be expected by chance.

The Poisson probabilities for the individual wards of Bulwell West and East were not statistically significant because of the arbitrary subdivision of large areas by the ward boundaries. This highlights one of the main disadvantages of probability mapping: some areas of high incidence may be missed because areas are divided haphazardly. The technique is relatively insensitive and will only detect substantial aggregations of SLE. It has previously been emphasised in an investigation of childhood cancer in the West Midlands, that probability mapping is a 'first level' tool of analysis and that other methods should be applied to data before final conclusions can be drawn. ${ }^{3}$ Nevertheless, it has the advantage of being a simple and quick technique that will highlight a very 'abnormal' pattern such as that seen with SLE cases in Nottingham.

With the use of a Poisson probability test, a number of false positive results may be expected by chance. ${ }^{326}$ However, our five wards with a Poisson probability of $p<0 \cdot 1$ seemed to aggregate into two areas (fig 1), the first comprising Carlton Hill, Lenton, Trent, and Bridge, and the second Bulwell West. Such aggregation of wards with a 'significant' Poisson probability makes it less likely that they have occurred by chance. ${ }^{27}$

Non-environmental factors could be responsible for an unusually large or small number of cases. There may be variation of General Practitioner referral patterns across the study area, which encompasses both recognition of diagnosis and demographic differences in the ward populations. However, comparison of population pyramids between individual wards and the whole study area suggests that age distribution does not account for the excess of cases seen in those wards with a significant $p$ value $(<0 \cdot 1)$. A number of wards demonstrated an absence of SLE cases despite having a population size that would have been predictive of at least one case, as calculated from the expected ward prevalence rates. Conversely, however, if environmental factors alone were responsible for SLE, one might argue that no cases should be the norm. Using Poisson probability testing, no statistical significance was demonstrated for any ward; however, it should be noted that the Poisson distribution curve is skewed to the left, and the resulting power to detect statistical significance in the wards with no cases is thus weak.

The calculated variogram of SLE incidence in Nottingham failed to confirm the discrete cluster suggested visually by the case map and by subsequent Poisson analysis. Variogram analysis may appear superior to probability mapping because it is not constrained by the borders of geographical wards and avoids sudden transitions between neighbouring areas. However, this technique is best suited for detecting spatial variation across a large area, rather than a local concentration of cases such as the 
apparent SLE cluster. Consequently, the cause(s) of the excess cases seen in Bulwell West, Bulwell East and other wards do not appear to have a discernible effect across the whole area.

The development of SLE may depend on a genetically susceptible individual being exposed to some environmental factor(s). However, as little is known about the incubation period of SLE, it is possible that such environmental factors may promote the expression of existing but previously dormant disease.

In conclusion, some areas in Nottingham have a higher than expected prevalence of SLE; however, current statistical methodologies, especially those adapted from non-medical spatial analyses, do not support the clustering seen on case mapping. Further investigations are in progress to define relevant local environmental factors and their possible role in disease causation.

The study was financed by Lupus UK, to whom we are indebted. We also wish to thank all our colleagues for their help with this study, and Mrs S Todd for assistance with computer analysis.

1 Snow J. On the mode of communication of cholera, 2nd edn. London: Churchill, 1855.

2 Burkitt D, Wright D. Burkitts lymphoma. Edinburgh: Livingstone, 1970

3 Muir K, Parkes S, Mann J, et al. 'Clustering'-real or apparent? Probability maps of childhood cancer in the West Midlands Health Authority Region. Int $\mathcal{F}$ Epidemio 1990; 19: 1-7.

4 Openshaw S, Craft A, Charlton M, Birch J. Investigation of leukaemia clusters by use of geographical analysis machine. Lancet 1988; i: 272-3.

5 Hopkinson N, Doherty M, Powell R. The prevalence and incidence of systemic lupus erythematosus, $U K$ 1989-90. Br f Rheumatol 1993; 32: 110-5.

6 Fielder A, Walport M, Batchelor J, et al. Family study of the major histocompatibility complex in patients with major histocoth of $\mathrm{C} 4 \mathrm{~A}$ and $\mathrm{C} 4 \mathrm{~B}$ in determining disease susceptibility. BMF 1983; 1: 425-8.
7 Deapen D, Weinrib L, Langholz B, Horvitz D, Mack T. A revised estimate of the twin concordance in SLE: A survey of 138 pairs. Arthritis Rheum 1986; 29: S26.

8 Siegel M, Lee S L. The epidemiology of systemic lupus erythematosus. Semin Arthritis Rheum 1973; 3: 1-54.

9 Fessel W J. Systemic lupus erythematosus in the community: incidence, prevalence, outcome and first symptoms; the high prevalence in black women. Arch Intern Med 1974; 134: 1027-35.

10 Morrison R C A, Gear A J, Kark A, Mevers A, Goldberg B. Differences in systemic lupus erythematosus among 4 racial groups in South Africa. Arthritis Rheum 1990; 33: S104.

11 Frank A. Apparent predisposition to systemic lupus erythematosus in Chinese patients in West Malaysia. Ann Rheum Dis 1980; 39: 266-9.

12 Serdula $M$, Rhoads $G$. Frequency of systemic lupus erythematosus in different ethnic groups in Hawaii. erythematosus in different ethnic
Arthritis Rheum 1979; 22: 328-33.

13 Lee S, Chase P. Drug induced systemic lupus erythematosus: a critical review. Semin Arthritis Rheum 1975; 5: 83-103.

14 Freni-Titulaer L, Kelley D, Grow A, et al. Clustering of connective tissue diseases (CTDs) in a small Georgia Community: III. A search for environmental factors. Arthritis Rheum 1988; 31: S75.

15 Freni-Titulaer L, Kelley D, Grow A, et al. Connective tissue disease in Southeastern Georgia: a case control study of aetiologic factors. Am 7 Epidemiol 1989; 130: 404-9.

16 Choynowski M. Maps based on probability. $7 \mathrm{Am}$ Stat Ass 1959; 54: 385-8.

17 Hopkinson N, Powell R. Screening of acute psychiatric admissions for previously undiagnosed systemic lupus erythematosus. Br f Psychiatry 1992; 191: 107-10.

18 Fries J, Holman H. Systemic lupus erythematosus. $A$ clinical analysis. Philadelphia: W B Saunders Co, 1976.

19 Tan E, Cohen A, Fries J, et al. The 1982 revised criteria for the classification of systemic lupus erythematosus. Arthritis Rheum 1982; 25: 1271-7.

20 Final mid-1988 OPCS estimates of resident population. Nottingham: Nottingham Health Authority, 1989.

211991 Census. City Report: Nottinghamshire (Part 1). London: OPCS, 1992.

22 Brazier S, Hammond $\mathrm{R}$, Waterman S, Brown $\mathrm{P}$. $A$ new geography of Nottingham. Nottingham: Trent Polytechnic,

23 Matheron G. Les variables regionalisées et leur estimation. Paris: Masson, 1965.

24 Matheron G. The theory of regionalized variables and its applications. Paris: Cahiers du Centre de Morphologie Mathematique de Fontainbleu, 1971.

25 Oliver M, Muir K, Webster R, et al. A geostatistical approach to the analysis of pattern in rare disease. $f \mathrm{Pub}$ Health Med 1992; 14: 280-9.

26 Wakeford R, Binks K, Wilkie D. Childhood leukaemia and nuclear installations. $\mathcal{F} R$ Statist $\operatorname{Soc} A$ 1989; 152: 61-86.

27 Barnes $\mathrm{N}$, Cartwright $\mathrm{R}, \mathrm{O}^{\prime} \mathrm{Brien} \mathrm{C}, \mathrm{A}$ al in electoral wards with high lympoterns in electoral wards with high lymphoma incidence in the 